\title{
Integração entre geradores fotovoltaicos e retrofit energético em edifícios
}

\author{
Integration between photovoltaic generators and building energetic retrofitting
}

\author{
Caio F. L. Munguba 1 (b) orcid.org/0000-0003-3524-2432 \\ Luis A. Gómez-Malagón 1 (D) orcid.org/0000-0002-5199-9390 \\ Gustavo de Novaes Pires Leite 2 (D) orcid.org/0000-0001-5147-3498 \\ Álvaro Antônio Villa Ochoa 2 (1) orcid.org/0000-0001-7597-3358 \\ ${ }^{1}$ Escola Politécnica de Pernambuco, Universidade de Pernambuco, Recife, Brasil,
${ }^{2}$ Instituto Federal de Pernambuco, Recife, Brasil.
E-mail do autor principal: Caio Munguba. Caiofmunguba@hotmail.com.br
Resumo
}

Cerca de $50 \%$ do consumo energético brasileiro corresponde aos edifícios, muitos considerados pouco eficientes e de concepção projetual ultrapassada. Contra isso, entidades governamentais têm estimulado por meio de normas e diretrizes os retrofits energéticos atrelados a geração distribuída, mirando os chamados ZEB, zero energy building. Retrofits energéticos podem reduzir em até $30 \%$ o consumo de um edifício existente, e junto à geração distribuída, podem possibilitar o alcance do $Z E B$, particularmente em edifícios educacionais. Neste sentido, explora-se a sugestão de um retrofit energético junto a instalação de um gerador fotovoltaico no bloco IK, um dos edifícios da Escola Politécnica de Pernambuco da Universidade de Pernambuco, considerando a autogeração, a eficiência do edifício e os parâmetros de conforto desejados sob indicadores econômicos. Embora complexa, a seleção das estratégias de retrofit pode ser justificada avaliando o edifício holisticamente a partir dos fundamentos do consumo, e através de um modelo que usando dados de mercado, seja capaz de indicar os panoramas energéticos e econômicos por trás dos cenários de intervenção propostos. Obteve-se que a autogeração justifica-se e que o retrofit é complementar ao gerador fotovoltaico, pois juntos, podem aumentar a razoabilidade do projeto se os indicadores econômicos e energéticos forem corretamente avaliados.

Palavras-Chave: Análise econômica; Climatização; Iluminação; Envoltória; Eficiência energética; Escola;

\begin{abstract}
Almost $50 \%$ of Brazilian power consumption is related with buildings, some of them evaluated as poorly efficient and outdated. In that way, government entities had been stimulating modernizations with guidelines and standards modernizations, proposing with retrofitting and solar power to reach ZEB (Zero Energy Building). Energetic retrofits can save about 30\% building power consumption, which with PV (Photovoltaic) generation turns reach ZEB easier, particularly to educational buildings. This paper explores the suggestion of an energetic retrofitting with PV generation to IK building of POLI-UPE (Pernambuco Polytechnic SchoolPernambuco University). Considering PV generation, building efficiency and comfort parameters under economic indicators. Despite the process complexities, it's possible to find a solution about strategies selection dilemma evaluating the building holistically from power consumption baselines, with a systematic methodology using market data that must be composed to figure out energetic and economic indicators beneath the proposed action backgrounds. The findings, overall seems that auto generation is fully reasonable and retrofit with, might affect positively the cost-saving if the intervention scenarios were rightly evaluated.
\end{abstract}

Key-words: Economic analysis; Cooling; Lightning; Housing; Energy efficiency; 


\section{Introdução}

Nos próximos dez anos, a demanda energética deve crescer em torno de $87 \%$ no Brasil, catalisado pelo crescimento da carga em edifícios novos e existentes, que juntos, já são $50 \%$ da energia consumida no Brasil [1]. Essa escalada interfere no comportamento da matriz energética, implicando em aumento da emissão de poluentes se não aliada ao uso de fontes renováveis.

Nesse contexto, projetos inadequados ao ambiente bioclimático podem ser apontados como uma das principais razões de uso irracional de energia na busca de parâmetros de conforto. Destarte, a arquitetura sustentável e bioclimática, baseada em materiais de alto desempenho e amigos do meio ambiente [2], levanta estratégias passivas que combinadas reduzem a pegada de carbono de edificações [3].

O retrofit, como ferramenta, consiste na atualização do edifício e seus sistemas [4], sendo retrofits energéticos relacionados ao aumento da eficiência energética e da performance do edifício [5]. Como estratégia para racionalizar o consumo e reduzir impactos, artifícios associados a arquitetura bioclimática e equipamentos eficientes são integrados [6]. E como produto, é possível minorar o consumo na ordem de $30 \%$ em edifícios existentes e $50 \%$ em novos [7].

Destarte, mitigar as cargas em edificações é um dos melhores caminhos para reduzir o custo operacional de sistemas energéticos [8]. E sobre este ponto, diretivas como a 2011/31/EU, relacionada ao desempenho energético de edifícios relata que novas edificações devem ser ZEB (Zero energy building) ou próximo até 2020 [9]; similarmente, DOE-USA (Department of energy) propõe que novas residências busquem ZEB até 2020 e edifícios comerciais até 2025 [10]. No Brasil, a resolução normativa SLTI/MP n²/2014 argumenta a favor da realização de licitações de sustentabilidade para aumentar a eficiência energética de edifícios e equipamentos públicos no âmbito dos programas de etiquetagem [11].

No Brasil, a geografia favorável e a regulamentação pelas resoluções ANEEL (Agência nacional de energia elétrica) n0482/2012 e n0687/2015 propiciam a instalação de geradores fotovoltaicos em edifícios. Esse tipo de gerador pode ser instalado na envolvente da edificação e 29 em cobertas independentemente do gradiente ou orientação [12], especialmente em universidades, onde comummente há espaços livres que podem ser usados sem implicações arquitetônicas [12].

Em prédios comerciais e educacionais, o uso energético está principalmente ligado a iluminação e climatização, considerados vilões do consumo [13]. Diante disso, a auditoria energética pode ser considerada o primeiro passo para alcançar ZEB [14], já que ponderando a complexidade do processo, a seleção das estratégias, deve visar primeiramente a redução do consumo e emissões [15], [16], passando pelo retrofit operacional e de equipamentos [17], [18], e pôr fim a aplicação da tecnologia das renováveis [19].

O bloco IK da UPE-POLI (Escola Politécnica de Pernambuco-Universidade de Pernambuco) [16], quando avaliado pelo sistema WebPrescritivo, ferramenta online de avaliação de eficiência de envoltórias de edificações comerciais pelos critérios do RTQ-C (Regulamento Técnico da Qualidade em Construções) desenvolvida pelo LABeee (Laboratório de Eficiência Energética em Edificações) [20], revela que o edifício possui fachadas e um sistema de iluminação artificial ambos ineficientes. Diante disso, se deveria estudar os sistemas de iluminação e condicionamento ativo e elaborar um estudo financeiro que incorpore as iniciativas em prol da eficiência energética [16].

Todavia, bons resultados estão relacionados a abordagem técnica do projeto, devido a variedade de estratégias e variáveis envolvidas, e ao fato de que muitas não se provam viáveis economicamente, chegando a ter pay-back próximo a 40 anos [21]. A questão principal então é como obter dentre as propostas, a melhor combinação, com melhores indicadores econômicos [22]. Observar o edifício holisticamente, considerando economicamente o sistema de climatização, iluminação e as intervenções de envoltória sob os indicadores econômicos juntos pode ser o melhor caminho.

\section{Materiais e métodos}

\subsection{Metodologia}

O programa de retrofit pode ter cinco etapas, como na figura 1 . Considerando as políticas regulamentarias, os recursos disponíveis, a edificação, fatores humanos, tecnologias e fatores 
desconhecidos. Todos visando aumento da eficiência energética e redução do uso de energia [16], [22].

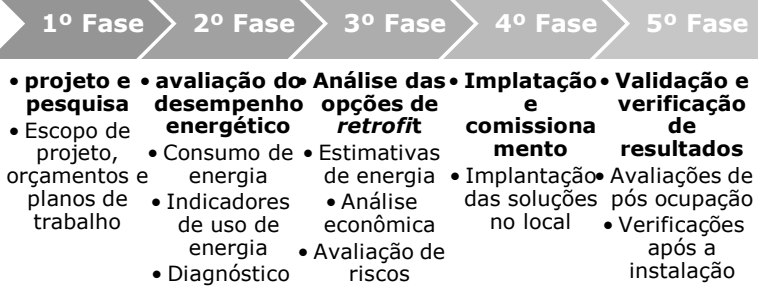

\begin{tabular}{|c|c|c|c|}
\hline $\begin{array}{c}1^{\circ} \text { Fase } \\
\text { Bloco IK e } \\
\text { POLI }\end{array}$ & $\begin{array}{l}2^{\circ} \text { Fase } \\
\text { Consumo e } \\
\text { medição }\end{array}$ & $\begin{array}{l}3^{\circ} \text { Fase } \\
\text { Cálculos e } \\
\text { dimensões }\end{array}$ & $\begin{array}{c}4^{\circ} \text { Fase } \\
\text { Indicadores } \\
\text { e resultados }\end{array}$ \\
\hline $\begin{array}{l}\text { - Dados } \\
\text { - Espaço/uso } \\
\text { - Cargas } \\
\text { - Térmica } \\
\text { - Carga } \\
\text { Lumínica } \\
\text { - Carga } \\
\text { Elétrica }\end{array}$ & $\begin{array}{c}\text { - Análise } \\
\text { - Panorama dos } \\
\text { dados atuais } \\
\text { - Tratamento } \\
\text { de dados } \\
\text { - Parâmetros de } \\
\text { conforto }\end{array}$ & $\begin{array}{l}\text { - Otimização } \\
\text { - Propostas de } \\
\text { redução de } \\
\text { carga } \\
\text { - Análise de } \\
\text { demanda } \\
\text { - Simulações }\end{array}$ & $\begin{array}{c}\text { - Financeiro } \\
\text { - Indicadores } \\
\text { • Cenários } \\
\text { • Comparações }\end{array}$ \\
\hline
\end{tabular}

FIgURA 1: Fases do retrofit. FonTE: Adaptado de Ma et al (2012).

O presente artigo foca nas fases $2^{\circ}$ e $3^{\circ}$ da metodologia proposta pelo grupo de pesquisa MA et al [22], como guia para justificar os resultados. Partindo da avaliação energética, passando pela análise de dados e chegando à otimização e as proposições, avaliados sob os indicadores econômicos.

FIgURA 2: Fases do presente artigo. FonTE: Dados dos autores.

\subsection{Bloco IK}

O bloco IK, edifício onde se aplicou a metodologia deste artigo, é um dos oito blocos da POLI-UPE, e possui $2.907,96 \mathrm{~m}^{2}$ ou $30 \%$ da área construída da escola. Destinado a pós-graduação e graduação, possui quatro pavimentos e 29 salas de aula, além de abrigar duas coordenações, uma sala de professores, uma sala de apoio ao estudante, uma sala de videoconferência, laboratórios, espaços de consultoria e oito banheiros [16].

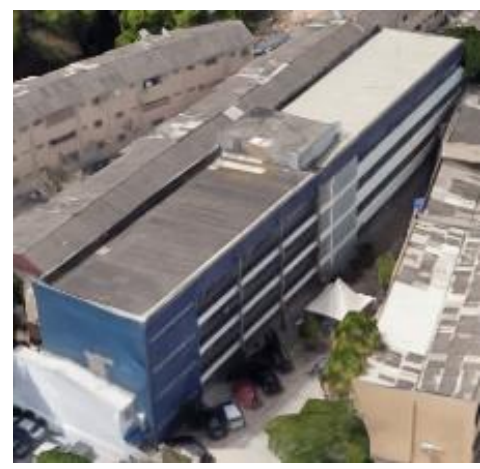

FIgURA 3: Bloco IK. FonTE: Google (2019).

A envoltória do bloco IK, constituída das vedações de periferia e coberta, avaliada pela ferramenta online WebPrescritivo, que ordena a eficiência entre $A$ e $E$, desponta como $E$, ineficiente devido a infiltração de calor pela coberta. Para alcançar A, foi proposto instalar forro de gesso no último pavimento, pintar as telhas com tinta reflexiva ou substituí-las por telhas sanduíche com poliestireno [16], conforme no quadro 1.

QUADRO 1: Melhorias propostas para envoltória nível A

\begin{tabular}{|c|c|}
\hline Melhorias & $\begin{array}{c}\text { Nova } \\
\text { eficiência }\end{array}$ \\
\hline Instalação de forro de gesso no $3^{\circ}$ pavimento & $\mathrm{C}$ \\
\hline $\begin{array}{c}\text { Instalação de forro de gesso no } 3^{\circ} \text { pavimento } \\
\text { e pintura da telha de fibrocimento }\end{array}$ & $\mathrm{B}$ \\
\hline $\begin{array}{c}\text { Instalação de forro de gesso no } 3^{\circ} \text { pavimento } \\
\text { e substituição de telha de fibrocimento por } \\
\text { poliestireno }\end{array}$ & $\mathrm{A}$ \\
\hline
\end{tabular}

FonTE: Adaptado de Barros (2018).

O sistema de iluminação artificial, avaliado pelo o critério do valor limite de DPIL (Densidade de Potência de Iluminação) [16], de 10,7 $\mathrm{W} / \mathrm{m}^{2}[23]$, atende ao nível $\mathrm{C}$ pelo método de simulação do WebPrescritivo. Para alcançar nível A e atender a NBR ISO/CIE 8995-1 [24], propôsse e modificar o layout das luminárias e os circuitos, substituindo as lâmpadas e buscando valores entre 500 e 550 lux por sala de aula com auxílio da luz natural, conforme figura 4 [16]. Neste novo layout, as luminárias destacadas em verde têm acionamento opcional, por causa da infiltração de luz natural pelas janelas da face sudeste do edifício. 


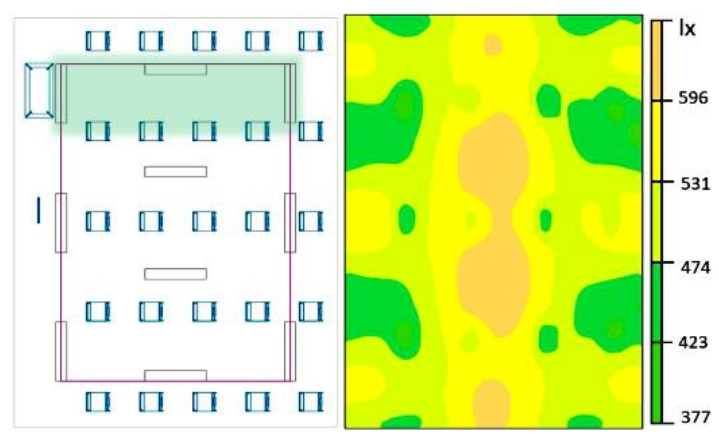

FIgURA 4: Novo layout de iluminação da sala de aula, com maior eficiência e considerando iluminação natural. FonTE: Adaptado de Barros (2018).

A carga operacional foi obtida do tratamento do inventário da escola, pesquisas em campo e dos horários de uso do primeiro semestre de 2019. A potência instalada em Watts está na figura 5 .

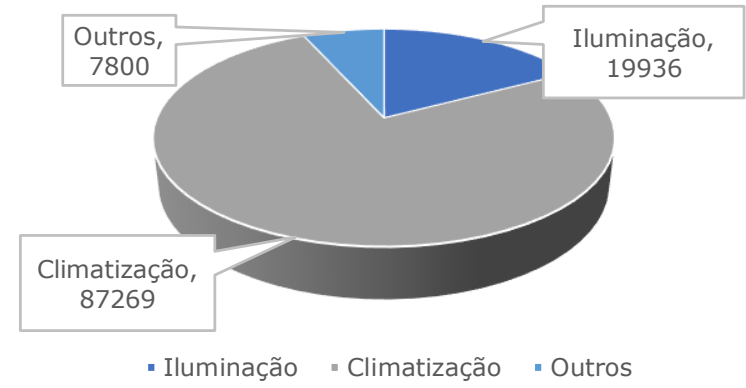

FIgURA 5: Potência instalada do bloco IK em W. FonTE: Dados dos autores.

\subsection{Consumo, medição e tarifa}

O consumo aferido da Escola é único para todos os blocos. Para encontrar a participação do bloco IK utilizou-se a metodologia disposta na figura 6 .

\begin{tabular}{|c|c|c|}
\hline $1^{\circ}$ Fase & $2^{\circ}$ Fase & $3^{\circ}$ Fase \\
\hline $\begin{array}{l}\text { - Levantamento } \\
\text { - Equipamentos } \\
\text { - Uso }\end{array}$ & $\begin{array}{l}\text { - Memória de } \\
\text { consumo da } \\
\text { POLI } \\
\text { - histórico de } \\
\text { medição da escola }\end{array}$ & $\begin{array}{l}\text { - Harmonização } \\
\text { - Obtenção da } \\
\text { memória de } \\
\text { consumo do bloco } \\
\text { IK }\end{array}$ \\
\hline
\end{tabular}

FIgURA 6: Metodologia para obtenção do consumo mensal do bloco IK. FonTE: Dados dos autores.

Com o inventário de equipamentos e do uso dos ambientes e a equação 1 [25] obteve-se o consumo das luminárias, ventiladores, projetores, computadores e demais equipamentos de escritório, assim:

$$
C=\frac{W \cdot H \cdot D}{1000}
$$

$C=$ Consumo em $\mathrm{kWh}$

$W=$ Potência instalada em Watts

$H=$ Horas de utilização

$D=$ Dias de utilização

Para equipamentos de climatização, temos [26].

$$
\text { Ehvac }=\text { Chvac.COP.UI.H }
$$

Onde:

Ehvac $=$ Consumo em kWh

Chvac $=$ Capacidade de refrigeração em BTU $/ \mathrm{h}$

$C O P=$ Coeficiente de performance em $[\mathrm{W} /(\mathrm{BTU} / \mathrm{h})]$

$U I=$ Índice de utilização, adotado 70\% [26].

$H=$ Número de horas

Sendo consumidor cativo do setor público alimentado em $2.3 \mathrm{kV}$ [16], o consumo é medido como grupo tarifário $A$, de estrutura binômia correspondente a energia ativa, em (kWh) e a demanda faturável em (kW) [27]. A Escola adota a tarifa horo-sazonal azul, em que a demanda, assim como o consumo, é ajustada em função do horário para a ponta, entre as 17:30 e 20:30 e fora ponta. A tarifa de demanda não foi usada nos indicadores econômicos deste trabalho.

A memória de consumo do bloco IK foi gerada com a superposição da memória de consumo da Escola, disponível na figura 7 com os dados da figura 8. A sazonalização foi feita pela multiplicação da variação sobre a média anual.

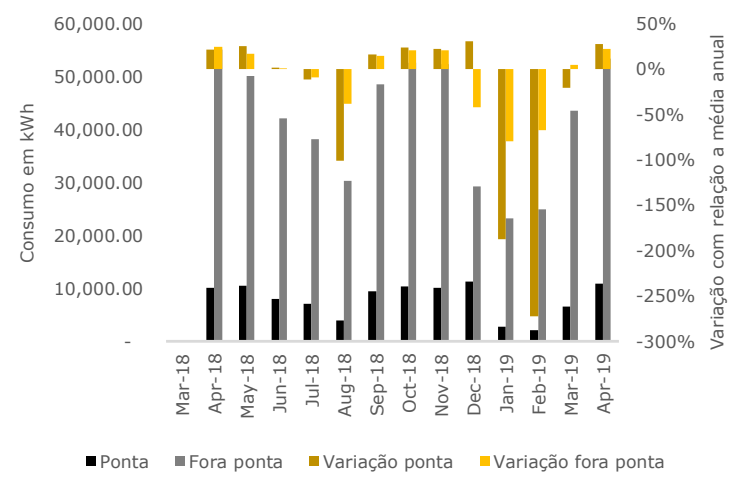

FIgURA 7: Memória de consumo da POLI com variação do consumo por mês e porcentagem com relação à média anual. FonTE: Dados dos autores.

Onde: 


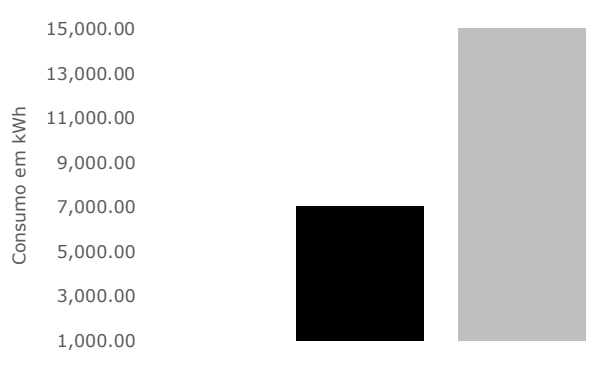

- Consumo Ponta = Consumo Fora Ponta

FIgURA 8: Consumo típico mensal do bloco IK considerando a média anual. FonTE: Dados dos autores.

O consumo sazonalizado em função do calendário anual encontra-se na figura 9. Convém lembrar que os meses de férias possuem menor consumo. O pico no consumo de ponta em dezembro é derivado do calendário de aulas da pós-graduação.

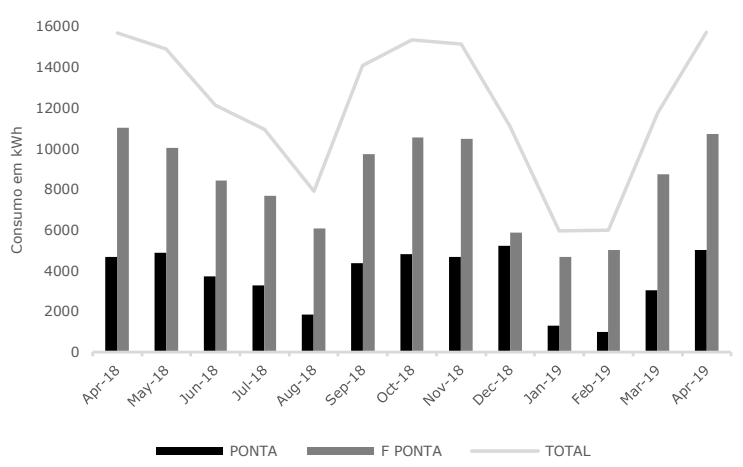

FIgURA 9: Consumo sazonalizado do bloco IK. FonTE: Dados dos autores.

\subsection{Calculo de carga térmica}

O cálculo de carga térmica foi realizado com o software Pro-Arcondicionado seguindo os critérios da NBR 16401-2 [28] e da NR 17 [29], e com os dados de ventilação, ocupação e iluminação expostos no quadro 2 .

QUADRo 2: Variáveis do cálculo da carga térmica dos ambientes.

\begin{tabular}{|c|c|}
\hline VARIÁVEL POR AMBIENTE & VALOR \\
\hline Altura teto/piso do ambiente & $3 \mathrm{~m}$ \\
\hline Área do ambiente & Variável \\
\hline Taxa de ocupação por pessoa no ambiente & $2 \mathrm{~m}^{2}$ \\
\hline Temperatura interna do ambiente & $23^{\circ} \mathrm{C}$ \\
\hline Temperatura de bulbo seco externa (15h) & $34^{\circ} \mathrm{C}$ \\
\hline Entalpia externa (15h) & $19.2 \mathrm{Kcal} / \mathrm{Kg}$ \\
\hline Umidade relativa do ar no ambiente & $50 \%$ \\
\hline Temperatura de bulbo seco (insuflamento) & $12{ }^{\circ} \mathrm{C}$ \\
\hline Taxa de iluminação do ambiente & $5 \mathrm{w} / \mathrm{m}^{2}(\mathrm{LED})$ \\
\hline Calor sensível liberado por pessoa & $68 \mathrm{Kcal} / \mathrm{h}$ \\
\hline
\end{tabular}

\begin{tabular}{|c|c|}
\hline Carga latente liberado por pessoa & $32 \mathrm{Kcal} / \mathrm{h}$ \\
\hline Outras cargas sensíveis do ambiente & $220 \mathrm{w}$ \\
\hline Variação temperatura ao longo do dia & $10^{\circ} \mathrm{C}$ \\
\hline
\end{tabular}
FONTE: Dados dos autores.

A climatização das salas de aula atualmente é realizada com máquinas do tipo Split, sem a renovação de ar requerida pela resolução Anvisa 09/2003 [30].

Os dados de transmitância da envoltória (U) (adimensional), foram obtidos da iniciativa ProjetEEE [31] e os de absortância (a) (adimensional), baseados em Dornelles et al [32], cujas informações estão expostas no quadro 3.

QUADRo 3: Transmitância (U) e absortância (a) das propostas.

\begin{tabular}{|c|c|c|c|}
\hline \multicolumn{2}{|c|}{ Situação } & $(\mathrm{U})$ & $(\mathrm{a})$ \\
\hline 1 & Coberta do 30 piso com forro de gesso & 1,3 & - \\
\hline 2 & $\begin{array}{c}\text { Coberta do } 3^{\circ} \text { piso com telhas de } \\
\text { poliestireno }\end{array}$ & 0,7 & - \\
\hline 3 & $\begin{array}{c}\text { Coberta do } 3^{\circ} \text { piso com telhas de } \\
\text { poliestireno e forro de gesso }\end{array}$ & 0,6 & - \\
\hline 4 & Fachada com cerâmica branca & - & Baixa \\
\hline
\end{tabular}

FonTE: Dados dos autores com base em ProjetEEE [31] e Dornelles et al [32].

Alcançar o nível A segundo o método WebPrescritivo foi o principal desafio para gerar cenários também baseados em Dornelles et al [33] e Lamberts et al [34], reduzindo a absortância das fachadas alterando a cor para branco, minimizando a infiltração de calor no telhado e instalando janelas de filmes para reduzir a penetração da luz efeito estufa.

\subsection{Dimensionamento do gerador fotovoltaico}

Para atingir o ZEB, o gerador fotovoltaico foi dimensionado pelo espaço $\left(684 \mathrm{~m}^{2}\right)$ e o consumo. Em primeiro lugar, a produção máxima foi obtida para o azimute do edifício $\left(120^{\circ}\right)$ e a inclinação do telhado $\left(5^{\circ}\right)$ com o PVsyst. Os dados solares do PVsyst estão expostos na figura 10.

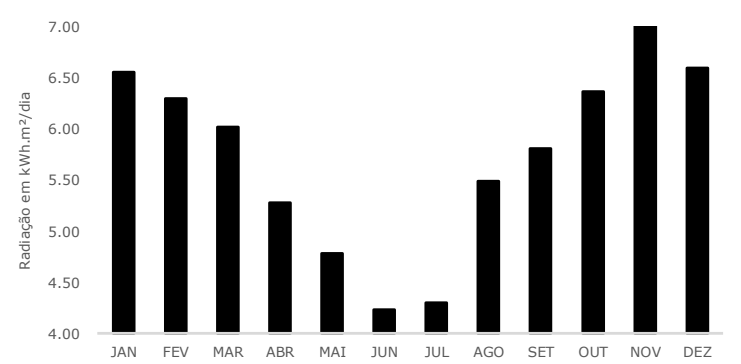

FIgURA 10: Radiação solar em $\mathrm{kWh} . \mathrm{m}^{2} / \mathrm{dia}$. FonTE: Dados dos autores.

DOI: $10.25286 /$ repa.v5i3.1268 
Com o ajuste tarifário [35] e os dados solares, o gerador fotovoltaico foi calculado para cada cenário de carga com a média de radiação anual, assim:

$$
P g f=\frac{\left(E f p+\left(\frac{E p}{(R \$ f p / R \$ p)}\right) / 30\right)}{I x \cdot(1-E g)}
$$

Onde:

$P g f$ = Potência do gerador fotovoltaico em kWp

$E f p=$ Energia consumida em $\mathrm{kWh}$ fora ponta em um mês típico

$E p$ = Energia consumida em $\mathrm{kWh}$ ponta em um mês típico

$R \$ f p=$ Valor cobrado por kWh fora ponta

$R \$ p=$ Valor cobrado por $\mathrm{kWh}$ ponta

$I$ = Média da radiação solar incidente em $\left(\mathrm{kWh} / \mathrm{m}^{2}\right)$. dia.

$E g=$ Perdas estimadas do gerador

O número 30 corresponde a um mês comercial.

Os dados gerados pelo PVsyst foram comparados com as saídas da equação 3 visando o ZEB. Os dados dos cálculos são mostrados no quadro 4.

QUADRo 4: Perdas consideradas no dimensionamento do gerador FV.

\begin{tabular}{|c|c|}
\hline PERDAS & VALOR \\
\hline Perdas por temperatura & $10 \%$ \\
\hline Perdas por mismatch & $2 \%$ \\
\hline Perdas por sujeira & $2 \%$ \\
\hline Perdas nos cabos CC & $1 \%$ \\
\hline Perdas nos cabos CA & $1 \%$ \\
\hline Perdas no inversor & $4 \%$ \\
\hline
\end{tabular}

FONTE: Autoria própria.

\subsection{Orçamentos e preços}

Para obter uma análise orçamentária realista, os preços dos equipamentos de climatização, iluminação e gerador fotovoltaico foram obtidos por pesquisa de mercado.

O custo dos equipamentos de climatização foi obtido em lojas virtuais, considerando tecnologia inverter e selo A de eficiência energética. Os preços estão na figura 11. Apenas preços médios foram utilizados.

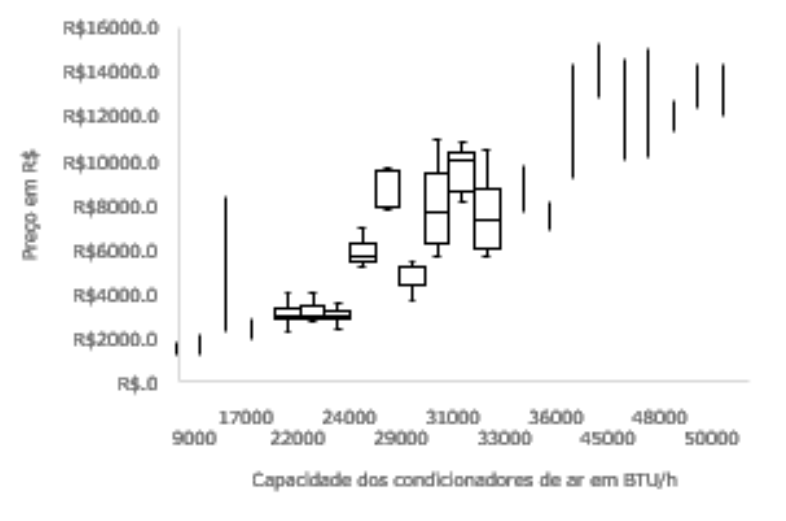

FIgURA 11: Preços dos aparelhos Split Inverter. FonTE: Dados dos autores.

Aos equipamentos de iluminação, embora da mesma forma, foi adicionado uma pesquisa local, considerando a tecnologia LED e o modelo T8 de 1,2 metros.

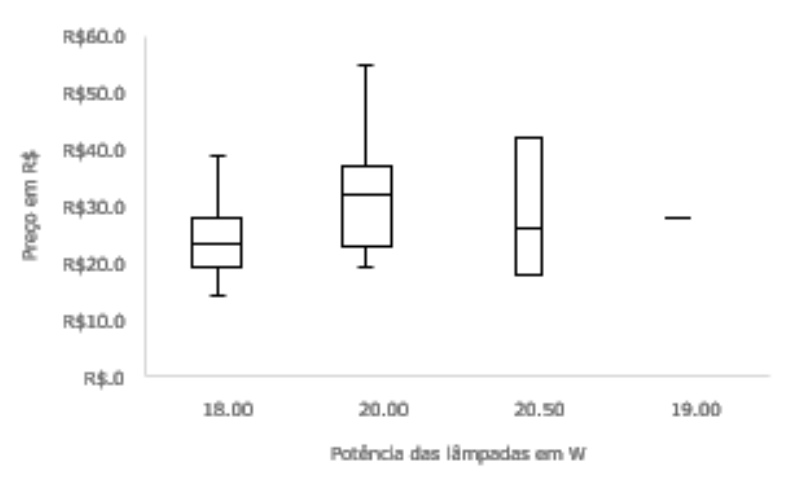

FigurA 12: Preço das lâmpadas tubulares LED. FonTE: Dados dos autores.

O preço dos geradores fotovoltaicos foi obtido por kits vendidos em lojas online. Esses kits contêm módulos, cabos, inversores e equipamentos de ancoragem. Apesar de não haver dois geradores fotovoltaicos iguais no mercado, por meio de regressão linear foi obtido o preço médio para cada potência máxima. Esse preço foi usado na equação 3 para obter os custos instalados do gerador fotovoltaico. Foi admitido $20 \%$ do preço do gerador como custos de instalação. Devido às mudanças de mercado, os modelos e marcas não foram discriminados. 


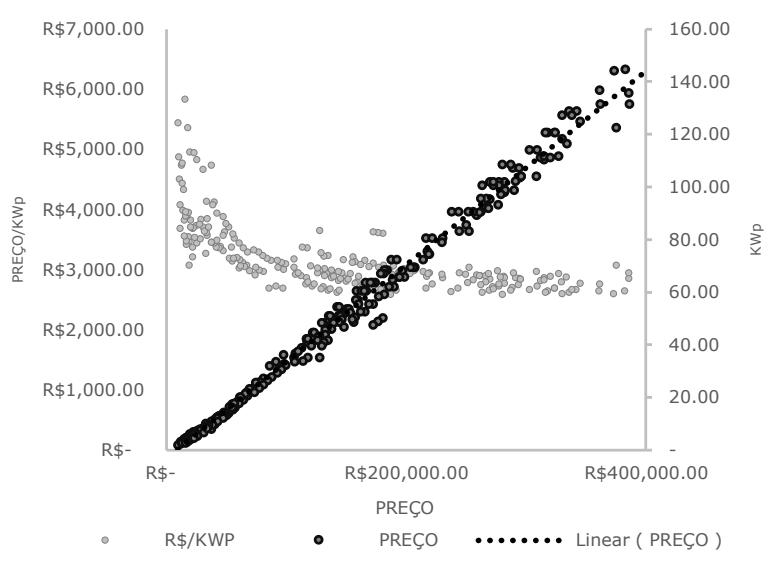

FIgURA 13: Custo do gerador em função da potência. FONTE: Dados dos autores.

Para obter os custos de retrofit de fachada foi usado o SINAPI (Sistema Nacional de Preços e Índices para a Construção Civil) não desonerado de abril de 2019 para composição dos preços, que também contém o material utilizado e projeção de lucro da ordem de $30 \%$ por serviço, simulando a eventual contratação de terceirizada. O preço dos materiais foi obtido de vendedores locais e online. Apenas o preço médio foi usado.

\subsection{Análise econômica}

Foram utilizados o fluxo de caixa (FC), o valor presente (VP), o valor presente líquido (VPL), a taxa interna de retorno (TIR), o índice de lucratividade (IL) e o pay-back para avaliar os cenários propostos [36]. Adotou-se 25 anos como vida útil e o reajuste tarifário, deterioração, manutenção expostos no quadro 5 .

QUADRo 5: Parâmetros da análise econômica.

\begin{tabular}{|c|c|c|c|}
\hline \multirow{2}{*}{ TIPO } & IDENTIFICAÇão & $\begin{array}{c}\text { PORCENTAGEM E AJUSTE } \\
\text { ANUAL }\end{array}$ & $\begin{array}{c}\text { VALOR } \\
\text { ATUAL }\end{array}$ \\
\hline \multirow{4}{*}{ Tarifa } & Ta p 06/2019 & $8 \%$ & 0.42 \\
\cline { 2 - 4 } & Ta fp 06/2019 & $8 \%$ & 0.27 \\
\cline { 2 - 3 } & Dem p 06/2019 & $8 \%$ & 40.84 \\
\cline { 2 - 3 } & Dem fp 06/2019 & $8 \%$ & 15.21 \\
\hline \multirow{3}{*}{ Ônus } & Manutenção & $1 \%$ & \multirow{1}{*}{} \\
\cline { 2 - 3 } & Inversores ano 15 & $15.00 \%$ & \\
\cline { 2 - 3 } & Degradação & $0.7 \%$ & \multicolumn{2}{|c}{} \\
\hline \multirow{2}{*}{$\$$} & Taxa desconto & $6 \%$ & \\
\hline
\end{tabular}

FONTE: Dados dos autores.

Adotou-se como taxa de desconto a taxa básica de juros definida pelo COPOM (Comitê de política monetária) para 2019 [37].

O fluxo de caixa (FC), conforme exposto no quadro 6, foi obtido descontando a energia produzida sobre a consumida [26].
QUADRo 6: Componentes do fluxo de caixa e seu papel.

\begin{tabular}{|c|c|}
\hline Investimento & Ônus \\
\hline Consumo & Ônus \\
\hline Geração & Bônus \\
\hline Manutenção & Ônus \\
\hline Inversores & Ônus \\
\hline Degradação & Ônus \\
\hline
\end{tabular}

FonTE: Dados dos autores.

$$
F C=\sum D R \$ n-\sum B R \$ n
$$

Em que:

$F C=$ Fluxo de caixa

$D R \$=$ Despesas do mês $n$

$B R \$=$ Bônus do mês $n$

O valor presente (VP), foi obtido do somatório de todos os fluxos de caixa.

$$
V P=\sum F C
$$

Em que:

$V P=$ Valor presente

$F C=$ Fluxo de caixa

O valor presente líquido (VPL), a estimativa do fluxo de caixa descontado no presente momento [38] usando uma taxa de desconto. Considerou-se o VPL nominal.

$$
V P L=-D R \$ 0+\sum_{n=1}^{1} \frac{D R \$}{(1-K)^{n}}
$$

Em que:

$V P L=$ Valor presente líquido

$D R \$ 0=$ Despesas no mês 0

$D R \$ n=$ Despesas no mês presente

$K=$ Taxa de desconto

$n=$ Mês presente

$O$ índice de lucratividade, que indica a capacidade de determinado investimento gerar retorno [39].

$$
I L=\frac{V P}{I 0}
$$

Em que:

$I L=$ Índice de lucratividade

$V P=$ Valor presente

$I 0$ = Investimento no mês 0

A taxa interna de retorno (TIR), é a taxa de desconto quando o (VPL) é igual a zero [26].

$$
T I R=K(\text { para } V P L=0)
$$

Em que: 
$T I R=$ Taxa interna de retorno

$K=\mathrm{TIR}$

$V P L=$ Valor presente líquido

O tempo de pay-back representa o período necessário par requerer todo o investimento [38].

$$
\sum_{n=0}^{P B D} R \$ n \geq 0
$$

Em que:

$P B D=$ Pay-back descontado

$R \$ n=$ Valor presente acumulado no momento $\mathrm{N}$.

\subsection{Cenários de intervenção}

Com os resultados de Barros [16] e os parâmetros da NR 17 [29], foi escolhido como cenário base o edifício totalmente climatizado com a instalação de aparelhos sem selo A e tecnologia inverter, como presentes hoje. Cada novo cenário foi composto com base nesta situação inicial, conforme figura 14 .

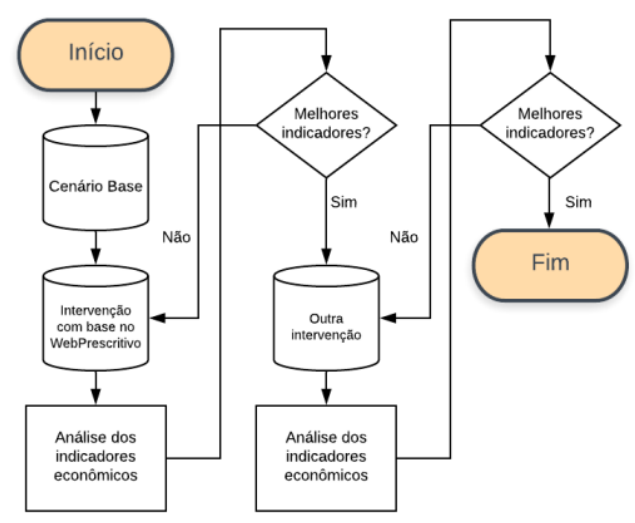

FIgURA 14: Metodologia de seleção de cenários. FoNTE: Dados dos autores.

Desde então, todo cenário foi gerado a partir do cenário base ou anterior de maior VPL. Logo quando melhores indicadores não são encontrados, o novo cenário é descartado. Quando obtidos, torna-se o novo cenário base.

Esses cenários foram classificados em seis grupos. No grupo $A$, o nível $A$ pelo WebPrescritivo foi buscado. Em B, foram testadas renovações de envelope não classificadas em PBE (Programa Brasileiro de Etiquetagem) -Edifica, como azulejos brancos em fachadas e filme em janelas. Além disso, o sistema de climatização foi modernizado com aparelhos inverter e selo A. Em C, primeiramente cada ambiente foi otimizado visando menor carga de energia e, posteriormente, menor consumo de energia. Em D, cada ambiente foi otimizado visando melhores TIR e IL para o projeto como um todo. Em E, todas as propostas de renovação foram usadas.

\section{Resultados e discussão}

\subsection{Cenários de intervenção}

Como mostrado no quadro 7, gerou-se 10 cenários para avaliar os possíveis resultados. Cenários de 1 a 6 estão relacionados com o grupo A e B. Cenários de 7 a 8 , para o grupo $C$, que vêm avaliar o peso do tempo de uso na relação cargaconsumo, ambos baseados no cenário 5 . O cenário 9, do grupo D, foi baseado em conhecimentos adquiridos ao longo das interações. O cenário 10, no final, compila todas as possibilidades.

QUADRO 7: Cenários gerados.

\begin{tabular}{|c|c|c|}
\hline \multicolumn{2}{|c|}{ Cenário } & Composição \\
\hline \multicolumn{2}{|c|}{ Base } & $\begin{array}{l}\text { Cenário sem intervenções de equipamento ou } \\
\text { envoltória, apenas instalação do gerador } \\
\text { fotovoltaico e climatização do } 2^{\circ} \text { e } 3^{\circ} \text { piso. Nível } \\
\text { (E) }\end{array}$ \\
\hline A & 1 & $\begin{array}{l}\text { Cenário base: Base. contém: climatização, } \\
\text { renovação dos circuitos de iluminação, } \\
\text { substituição das lâmpadas. nível (e) }\end{array}$ \\
\hline A & 2 & $\begin{array}{l}\text { Cenário base: Cenário 1. contém: climatização, } \\
\text { renovação dos circuitos de iluminação, } \\
\text { substituição das lâmpadas, forro de gesso no } \\
3^{\circ} \text { piso. nível (c) }\end{array}$ \\
\hline A & 3 & $\begin{array}{l}\text { Cenário base: Cenário 1. contém: climatização, } \\
\text { renovação dos circuitos de iluminação, } \\
\text { substituição das lâmpadas, forro de gesso no } \\
3^{\circ} \text { piso, telhas de poliuretano. nível (a) }\end{array}$ \\
\hline B & 4 & $\begin{array}{l}\text { Cenário base: Cenário 1. contém: climatização, } \\
\text { renovação dos circuitos de iluminação, } \\
\text { substituição das lâmpadas, substituição por } \\
\text { fachada branca. }\end{array}$ \\
\hline B & 5 & $\begin{array}{l}\text { Cenário base: Cenário 1. contém: climatização, } \\
\text { renovação dos circuitos de iluminação, } \\
\text { substituição das lâmpadas, instalação de } \\
\text { películas. }\end{array}$ \\
\hline B & 6 & $\begin{array}{l}\text { Cenário base: Cenário 1. contém: climatização, } \\
\text { renovação dos circuitos de iluminação, } \\
\text { substituição das lâmpadas, instalação de } \\
\text { películas, substituição e instalação de aparelhos } \\
\text { inverter. }\end{array}$ \\
\hline C & 7 & $\begin{array}{l}\text { Cenário base: Cenário 5. contém: variadas } \\
\text { soluções por ambiente, buscando menor } \\
\text { potência instalada por sala. }\end{array}$ \\
\hline C & 8 & $\begin{array}{l}\text { Cenário base: Cenário 5. contém: variadas } \\
\text { soluções por ambiente, buscando menor } \\
\text { consumo em kWh por sala. }\end{array}$ \\
\hline $\mathrm{D}$ & 9 & $\begin{array}{l}\text { Cenário base: Cenário } 5 \text {. contém: variadas } \\
\text { soluções por ambiente buscando melhores } \\
\text { indicadores econômicos. soluções: substituição } \\
\text { das lâmpadas, instalação de películas, } \\
\text { substituição do ar condicionado da sala I4. }\end{array}$ \\
\hline$E$ & 10 & $\begin{array}{l}\text { Cenário base: Base. contém: todas as } \\
\text { intervenções propostas. }\end{array}$ \\
\hline
\end{tabular}

FonTE: Dados dos autores. 


\subsection{Carga e consumo}

Com base no consumo de energia, os cenários no quadro 8 mostram a relação contra intuitiva entre o consumo de energia e a carga instalada, afetada pelo tempo de uso. A cor diz de verde para vermelho, melhor para o pior comportamento.

QUADRO 8: Resultados de carga em W e consumo anual em kWh.

\begin{tabular}{|c|c|c|c|c|c|}
\hline $\begin{array}{c}\text { CEN } \\
\text { ÁRI } \\
\mathbf{O}\end{array}$ & $\begin{array}{c}\text { CARGA } \\
\mathbf{K W}\end{array}$ & $\begin{array}{c}\text { CUSTO DA } \\
\text { INTERVENÇÃo }\end{array}$ & $\begin{array}{c}\text { DEMANDA } \\
\text { TÉRMICA } \\
\text { BTU/H }\end{array}$ & $\begin{array}{c}\text { CONSUMO } \\
\text { ANUAL } \\
\text { MWH }\end{array}$ & $\begin{array}{c}\text { CONSUMO } \\
\text { ANUAL R\$ }\end{array}$ \\
\hline 10 & 143.02 & $\mathrm{R} \$ 511.922 .82$ & 1.263 .966 .70 & 197.26 & $\mathrm{R} \$ 61.931 .61$ \\
\hline 8 & 141.35 & $\mathrm{R} \$ 395.599 .62$ & 1.286 .479 .10 & 197.44 & $\mathrm{R} \$ 62.106 .06$ \\
\hline 6 & 144.08 & $\mathrm{R} \$ 417.796 .39$ & 1.286 .479 .10 & 199.18 & $\mathrm{R} \$ 62.582 .47$ \\
\hline 7 & 128.16 & $\mathrm{R} \$ 354.757 .09$ & 1.286 .479 .10 & 202.16 & $\mathrm{R} \$ 63.487 .89$ \\
\hline 9 & 157.59 & $\mathrm{R} \$ 192.902 .27$ & 1.286 .479 .10 & 215.55 & $\mathrm{R} \$ 67.793 .73$ \\
\hline 5 & 169.76 & $\mathrm{R} \$ 178.935 .93$ & 1.286 .479 .10 & 226.93 & $\mathrm{R} \$ 72.088 .35$ \\
\hline 3 & 169.76 & $\mathrm{R} \$ 234.240 .74$ & 1.418 .323 .80 & 246.19 & $\mathrm{R} \$ 78.149 .09$ \\
\hline 2 & 169.76 & $\mathrm{R} \$ 158.534 .11$ & 1.434 .040 .80 & 247.75 & $\mathrm{R} \$ 78.669 .70$ \\
\hline 4 & 169.76 & $\mathrm{R} \$ 151.399 .45$ & 1.435 .471 .10 & 247.88 & $\mathrm{R} \$ 78.714 .75$ \\
\hline 1 & 169.76 & $\mathrm{R} \$ 145.069 .34$ & 1.435 .786 .90 & 247.92 & $\mathrm{R} \$ 78.727 .54$ \\
\hline Base & 180.57 & $\mathrm{R} \$ 125.234 .00$ & 1.442 .429 .20 & 261.33 & $\mathrm{R} \$ 82.883 .77$ \\
\hline
\end{tabular}

FONTE: Dados dos autores.

Conforme figura 15 , o cenário 10 , tem o melhor consumo mesmo não sendo a menor potência instalada por causa do COP (Coefficient of Performance) dos equipamentos de climatização, assim como o cenário 6 . Comparando as metodologias do grupo $\mathrm{C}$, o cenário 8 tem melhores resultados do que o cenário 7, devido a manutenção dos equipamentos de climatização. Avaliando as soluções de envelope, o cenário 5 foi melhor. Comparando o cenário base e o 10, cerca de 15 mil kWh foram salvos em um ano.

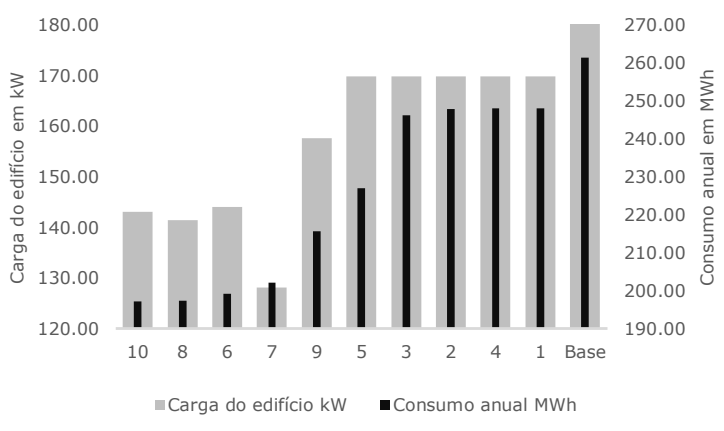

FIgURA 15: Potência e consumo da edificação. FonTE: Dados dos autores.

A figura 16 indica que o aumento do COP da climatização, apesar dos bons resultados em eficiência, pode custar muito. Como nos cenários 10 e 6 , os custos de renovação argumentam contra esse tipo de solução. Do outro lado, o cenário 1 mostrou-se razoável.

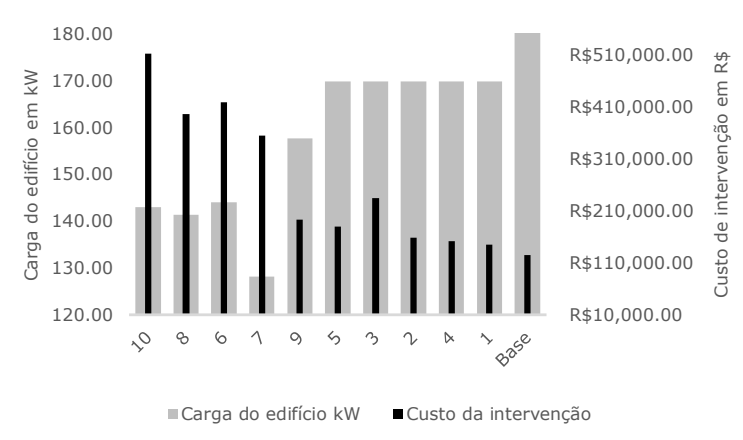

FIgURA 16: Potência e custo de intervenção. FonTE: Dados dos autores.

No lado direito das figuras 15 e 16, o cenário base exige menos capital, porém o consumo de energia reduz seu custo-benefício ao longo dos anos. Por outro lado, o cenário 10 implica que mais capital não significa melhores resultados porque, apesar de seus ganhos energéticos, não foram encontrados melhores indicadores econômicos.

\subsection{Gerador e geração}

Dimensionado com base nas equações 1 e 2 , o gerador está diretamente relacionado com o consumo de energia, como mostrado no quadro 9. A cor diz de verde para vermelho, melhor para pior comportamento.

QUADRo 9: Resultados de gerador e geração anual em kWh.

\begin{tabular}{|c|c|c|c|c|}
\hline CENÁRIO & $\begin{array}{c}\text { CUSTO DO } \\
\text { GERADOR }\end{array}$ & $\begin{array}{c}\text { GERADOR } \\
\text { KWPP }\end{array}$ & $\begin{array}{c}\text { GERAÇÃo } \\
\text { ANUAL MWH }\end{array}$ & $\begin{array}{c}\text { GERAÇÃO ANUAL } \\
\text { R\$ }\end{array}$ \\
\hline 9 & $\mathrm{R} \$ 465.780 .65$ & 152.13 & 251.09 & $\mathrm{R} \$ 67.793 .73$ \\
\hline 5 & $\mathrm{R} \$ 494.692 .25$ & 161.77 & 266.99 & $\mathrm{R} \$ 72.088 .35$ \\
\hline 1 & $\mathrm{R} \$ 539.387 .58$ & 176.67 & 291.58 & $\mathrm{R} \$ 78.727 .54$ \\
\hline 4 & $\mathrm{R} \$ 539.301 .53$ & 176.64 & 291.54 & $\mathrm{R} \$ 78.714 .75$ \\
\hline Base & $\mathrm{R} \$ 567.367 .53$ & 185.99 & 306.98 & $\mathrm{R} \$ 82.883 .77$ \\
\hline 2 & $\mathrm{R} \$ 538.998 .20$ & 176.54 & 291.37 & $\mathrm{R} \$ 78.669 .70$ \\
\hline 3 & $\mathrm{R} \$ 535.493 .45$ & 175.37 & 289.44 & $\mathrm{R} \$ 78.149 .09$ \\
\hline 7 & $\mathrm{R} \$ 436.793 .55$ & 142.47 & 235.14 & $\mathrm{R} \$ 63.487 .89$ \\
\hline 8 & $\mathrm{R} \$ 427.490 .97$ & 139.37 & 230.02 & $\mathrm{R} \$ 62.106 .06$ \\
\hline 6 & $\mathrm{R} \$ 430.698 .19$ & 140.44 & 231.79 & $\mathrm{R} \$ 62.582 .47$ \\
\hline 10 & $\mathrm{R} \$ 426.316 .60$ & 138.98 & 229.38 & $\mathrm{R} \$ 61.931 .61$ \\
\hline
\end{tabular}

FoNTE: Dados dos autores.

O gerador e as intervenções face a face estão expostos na figura 17 . Obtém-se que a intervenção e os custos geradores são aparentemente desconectados, mas complementares, conforme revelado no quadro 9. Observa-se também que os cenários 9,5,1 e 4 têm 
custos totais mais baixos do que o cenário de base, embora requeiram mais investimento no gerador. Quando os custos de renovação são maiores que os custos do gerador fotovoltaico, por outro lado, sacrifica-se a razoabilidade financeira do projeto.

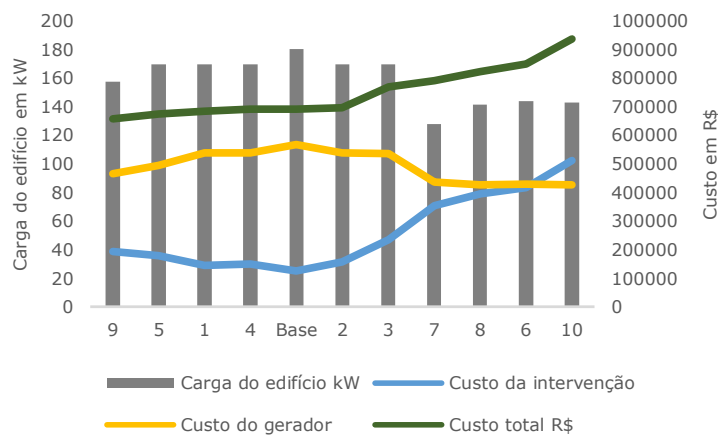

FIgURA 17: Custo de gerador e intervenção com potência e consumo. FonTE: Dados dos autores.

\subsection{Indicadores econômicos}

Classificados pelo VPL, no quadro 10 as cores dizem de verde para vermelho, melhor para pior performance.

QUADRo 10: Resultado dos indicadores econômicos.

\begin{tabular}{|c|c|c|c|c|c|}
\hline Cenário & Custo total $\mathrm{R} \$$ & $\mathrm{VPL}$ & $\mathrm{IL}$ & $\mathrm{TIR}$ & PAY-BACK \\
\hline 9 & $\mathrm{R} \$ 658.682 .91$ & $\mathrm{R} \$ 1.900 .077 .80$ & 3.88 & $21 \%$ & 7 anos e 2 meses \\
\hline 5 & $\mathrm{R} \$ 673.628 .19$ & $\mathrm{R} \$ 1.882 .427 .32$ & 3.79 & $20 \%$ & 7 anos e 3 meses \\
\hline 1 & $\mathrm{R} \$ 684.456 .92$ & $\mathrm{R} \$ 1.867 .416 .54$ & 3.73 & $20 \%$ & 7 anos e 3 meses \\
\hline 4 & $\mathrm{R} \$ 690.700 .98$ & $\mathrm{R} \$ 1.861 .180 .54$ & 3.69 & $20 \%$ & 7 anos e 3 meses \\
\hline Base & $\mathrm{R} \$ 692.601 .53$ & $\mathrm{R} \$ 1.856 .653 .91$ & 3.68 & $20 \%$ & 7 anos e 3 meses \\
\hline 2 & $\mathrm{R} \$ 697.532 .31$ & $\mathrm{R} \$ 1.854 .377 .59$ & 3.66 & $20 \%$ & 7 anos e 3 meses \\
\hline 3 & $\mathrm{R} \$ 769.734 .19$ & $\mathrm{R} \$ 1.782 .503 .64$ & 3.32 & $18 \%$ & 8 anos e 3 meses \\
\hline 7 & $\mathrm{R} \$ 791.550 .64$ & $\mathrm{R} \$ 1.769 .922 .34$ & 3.24 & $18 \%$ & 8 anos e 3 meses \\
\hline 8 & $\mathrm{R} \$ 823.090 .59$ & $\mathrm{R} \$ 1.739 .252 .80$ & 3.11 & $17 \%$ & 9 anos e 3 meses \\
\hline 6 & $\mathrm{R} \$ 848.494 .58$ & $\mathrm{R} \$ 1.713 .548 .72$ & 3.02 & $17 \%$ & 9 anos e 3 meses \\
\hline 10 & $\mathrm{R} \$ 938.239 .41$ & $\mathrm{R} \$ 1.624 .213 .86$ & 2.73 & $16 \%$ & 10 anos e 4 meses \\
\hline
\end{tabular}

FONTE: Dados dos autores.

Os indicadores estão ligados aos custos iniciais, portanto, os cenários de menor custo total estornam melhores indicadores, em destaque, maior VPL. Observa-se que o cenário 9 embora não possua os menores custos de renovação, gerador, consumo ou carga instalada, estorna melhor VPL, argumentando tem prol da auditoria energética e avaliação do projeto sob os indicadores econômicos. Comparando o cenário 9 e o 10 , salvam-se $R \$ 250$ mil ao longo da vida útil do projeto. Obtém-se também que os cenários com custos totais maiores que do cenário base, devem ser rejeitados.
A figura 18 expõe o VPL em 25 anos para os cenários 9, de melhor desempenho, 10, de pior desempenho e base.

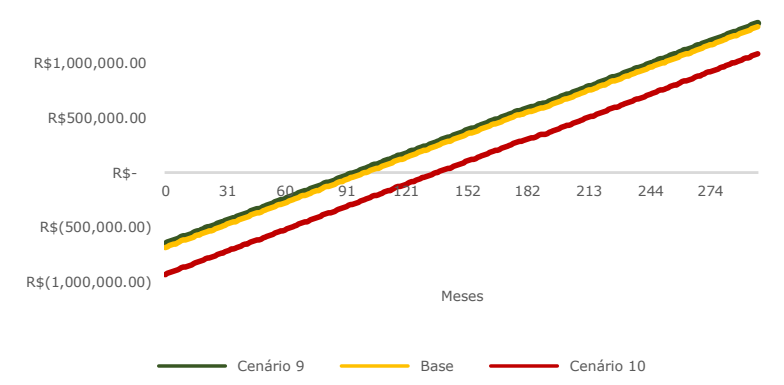

FIgURA 18: VPL em 25 anos. FonTE: Dados dos autores.

Assim sendo, os resultados principais deste trabalho são:

1. O VPL positivo em todos cenários indica retorno maior que o investimento inicial ao fim do prazo de 25 anos.

2. O tempo de retorno para cada cenário gira em torno de 87 e 136 meses, então entre 30 a $40 \%$ da vida útil estipulada.

3. Os indicadores econômicos argumentam que selecionar as intervenções mais eficazes é essencial se considerada a incorporação de um retrofit ao projeto, mas o cenário base também se mostrou viável economicamente.

4. Não é possível chegar ao ZEB com o espaço disponível e as cargas mesmo após as intervenções propostas.

5. A otimização do COP do sistema de climatização tem significativo impacto no consumo do edifício, porém os custos envolvidos contra-argumentam a esta estratégia.

6. Obteve-se que buscar menor consumo ou potência não aparentaram ser boas estratégias por não estornarem o maior VPL.

7. Soluções isoladas podem ter grande impacto positivo no projeto, pois o cenário 9 difere do 5 apenas na substituição de um aparelho de climatização.

8. O cenário 9, cujas soluções estão disponíveis no quadro 7 , estornou os melhores indicadores econômicos. 


\section{Conclusões}

Foram explorados os custos relacionados a instalação de geradores fotovoltaicos em harmonia com retrofits energéticos visando melhores indicadores econômicos.

As conclusões principais deste trabalho são:

1. A harmonização entre a renovação de uma edificação para eficiência energética e a instalação de um gerador fotovoltaico é um processo complexo que envolve dezenas de variáveis e ciências diferentes.

2. Se não for devidamente avaliado, há risco de obter efeito oposto ao esperado com o retrofit, aumentando os custos e reduzindo o desempenho dos indicadores econômicos.

3. Pode ser ineficaz alcançar os parâmetros de eficiência do PBE-Edifica.

4. As principais variáveis foram o orçamento da renovação e o consumo global da edificação, que sendo independentes, complementam-se e definem a análise econômica.

5. Auditoria energética com análise de retrofit antes do dimensionamento do gerador fotovoltaico, incorporando estratégias de condicionamento e iluminação ao projeto, mostrou-se eficaz, logo, encoraja-se a realização.

Os resultados deste trabalho podem contribuir para compreensão da harmonização do retrofit energético em edifícios e a instalação de geradores fotovoltaicos, por fundamentar-se em dados reais e comparar soluções pela proposição de cenários de intervenção.

\section{Referências}

[1] Ministério de Minas e Energia, "Brazilian National Energy Efficiency Plan - Premises and Guidelines," Princ. Guidel., p. 156, 2011.

[2] O. Orbella e S. Yannas, "Em busca de uma arquitetura sustentavel para os tropicos." p. $308,2009$.

[3] E. H. Mathews, C. P. Botha, D. C. Arndt, e A. Malan, "HVAC control strategies to enhance comfort and minimise energy usage," Energy Build., vol. 33, no. 8, pp. 853-863, 2001.

[4] ABNT, "ABNT NBR 15575:2013, Desempenho de edificações habitacionais," Associação Brasileira de Normas Técnicas, 2013. .

[5] M. Rabani, H. B. Madessa, e N. Nord, "A stateof-art review of retrofit interventions in buildings towards nearly zero energy level," Energy
Procedia, vol. 134, no. 1876, pp. 317-326, 2017.

[6] R. Fossati, Michele; Lamberts, "Eficiência energética da envoltória de edifícios de escritórios de Florianópolis: discussões sobre a aplicação do método prescritivo do RTQ-C Energy efficiency of office buildings envelope: discussions around application of prescriptive method of RTQ-C," vol. 10, no. 2, pp. 59-69, 2010.

[7] Tiago Reis, "Selo Procel Edificações é mais uma ferramenta para a eficiência energética." Disponível:

http://www.procelinfo.com.br/main.asp?ViewID =\%7B8D1AC2E8-F790-4B7E-8DDD-

CAF4CDD2BC34\%7D\&params $=$ itemID $=\% 7 B F 2$ 2EAB50-9E65-4500-92CC-

C50B26307A7E\%7D;\&UIPartUID =\%7BD90F22 DB-05D4-4644-A8F2-FAD4803C8898\%7D.

Acesso: 21-Jun-2019.

[8] J. Kneifel, "Life-cycle carbon and cost analysis of energy efficiency measures in new commercial buildings," Energy Build., vol. 42, no. 3, pp. 333-340, 2010.

[9] Parlamento Europeu, "Directiva 2010/31/UE (reformulação)," J. Of. da União Eur., pp. 13-35, 2010.

[10] C. Z. Net, E. Home, and S. Jose, "DOE One Sky Homes," pp. 0-3.

[11] "INSTRUÇÃO NORMATIVA N 2, DE 04 DE JUNHO DE 2014." Disponível: https://www.comprasgovernamentais.gov.br/in dex.php/legislacao/instrucoes-normativas/304instrucao-normativa-n-2-de-04-de-junho-de2014. Acesso: 21-Jun-2019.

[12] C. Peng, Y. Huang, and Z. Wu, "Buildingintegrated photovoltaics (BIPV) in architectural design in China," Energy Build., vol. 43, no. 12, pp. 3592-3598, 2011.

[13] J. C. S. Gonçalves and D. H. S. Duarte, "Arquitetura sustentável: uma integração entre ambiente, projeto e tecnologia em experiências de pesquisa, prática e ensino," Ambient. Construído, vol. 6, no. 4, pp. 51-81, 2006.

[14] P. A. Torcellini and D. B. Crawley, "Understanding zero-energy buildings," ASHRAE J., vol. 48, no. 9, 2006.

[15] R. M. Serafin, "Avaliação da redução do consumo de energia elétrica em funçao do retrofit no edifício sede da eletrosul," p. 139, 2010.

[16] M. L. Barros de Sousa Brito Perreira, "Diagnóstico e proposição de alternativas para eficiência energética em instituições de ensino: Estudo de caso na escola politécnica de Pernambuco," Universidade de Pernambuco, 2018.

[17] S. E. Chidiac, E. J. C. Catania, E. Morofsky,e S. Foo, "A screening methodology for implementing cost effective energy retrofit measures in Canadian office buildings," Energy Build., vol. 43, no. 2-3, pp. 614-620, 2011.

[18] M. D. Silva Junio, "Análise de soluções passivas em climatização via simulação computacional de estudo de caso," p. 140, 2013.

[19] A. Jafari e V. Valentin, "Selection of optimization objectives for decision-making in building

DOI: $10.25286 /$ repa.v5i3.1268 
energy retrofits," Build. Environ., vol. 130, no. September 2017, pp. 94-103, 2018.

[20] "WebPrescritivo - Etiquetagem de Edificações Comerciais, Públicas e de Serviços." Disponível: http://www.labeee.ufsc.br/sites/default/files/w ebprescritivo/index.html. Acesso: 21-Jun-2019.

[21] X. Sun, Z. Gou, e S. S. Y. Lau, "Costeffectiveness of active and passive design strategies for existing building retrofits in tropical climate: Case study of a zero energy building," J. Clean. Prod., vol. 183, pp. 35-45, 2018.

[22] Z. Ma, P. Cooper, D. Daly, e L. Ledo, "Existing building retrofits: Methodology and state-of-theart," Energy Build., vol. 55, pp. 889-902, 2012.

[23] INMETRO, "Regulamento Técnico da Qualidade para o Nível de Eficiência Energética de Edificações Comerciais, de Serviços e Públicas.," Brasília, 2013.

[24] ABNT, "Nbr Iso/Cie 8995-1," Assoc. Bras. Normas Técnicas, p. 46, 2013.

[25] CELPE, "Portal de Serviços da Celpe - Conheça sua Conta," 2019.2 Disponível: http://servicos.celpe.com.br/residencialrural/Pages/Baixa Tensão/conheca-suaconta.aspx. Acesso: 18-Jun-2019.

[26] G. de Novaes Pires Leite, F. Weschenfelder, A. M. Araújo, Á. A. Villa Ochoa, N. da Franca Prestrelo Neto, e A. Kraj, "An economic analysis of the integration between air-conditioning and solar photovoltaic systems," Energy Convers. Manag., vol. 185, no. November 2018, pp. 836849, 2019.

[27] Eletrobras; Procel; Procel edifica, "Manual de tarifação da energia elétrica," Rio de Janeiro, 2011.

[28] ABNT, "NBR 16401-2 Instalações de arcondicionado - Sistemas centrais e unitários. Parte 2: Parâmetros de conforto térmico," Rio Janeiro/RJ, pp. 1-79, 2008.

[29] Ministério do trabalho e emprego, "Norma regulamentadora 17, ergonomia," 2007. Disponível:

http://trabalho.gov.br/images/Documentos/SS T/NR/NR17.pdf. Acesso: 18-Jun-2019.

[30] Anvisa, "Ministério da Saúde - Resolução 09/2003," 2003.

[31] INMETRO, "Anexo geral V-Catálogo de propriedades térmicas de paredes, coberturas e vidros," 2017.

[32] Projeteee, "Componentes Construtivos | ProjetEEE." Disponível: http://projeteee.mma.gov.br/componentesconstrutivos/. Acesso: 18-Jun-2019.

[33] K. A. Dornelles, M. Roriz, E. K. A. Dornelles, e P. M. Roriz, "Influência das tintas imobiliárias sobre o desempenho térmico e energetico de edificações," $X$ Congr. Int. Tintas, 2007.

[34] lambert, "Desempenho térmico em edificações," Univ. Fed. St. Catarina, vol. 70, p. 239, 2016.

[35] V. Ayrão, "Como calcular FV para clientes do Grupo A," 2016.2 Disponível: https://viniciusayrao.com.br/como-calcular-fvpara-clientes-do-grupo-a/. Acesso: 18-Jun2019.

[36] F. S. Westphal e R. Lamberts, "Estudo de viabilidade econômica de uma proposta de retrofit em um edifício comercial," V Encontro
Nac. Conforto no Ambient. e II Encontro LatinoAmericano Conforto no Ambient. Construído, no. January, p. 9, 1999.

[37] Banco Central do Brasil, "Taxas de juros básicas - Histórico," 2019.2 Disponível: https://www.bcb.gov.br/controleinflacao/histori cotaxasjuros. Acesso: 31-Jul-2019.

[38] R. Villena-Ruiz, F. J. Ramirez, A. HonrubiaEscribano, e E. Gómez-Lázaro, "A technoeconomic analysis of a real wind farm repowering experience: The Malpica case," Energy Convers. Manag., vol. 172, pp. 182-199, Sep. 2018.

[39] Voitto, "Índice de Lucratividade: o que é e como calcular | Blog Voitto." Disponível: https://www.voitto.com.br/blog/artigo/indicede-lucratividade. Acesso: 18-Jun-2019. 